\title{
The Counting of Generalized Polarizabilities
}

\author{
Richard F. Lebed \\ Department of Physics and Astronomy, \\ Arizona State University, Tempe, Arizona 85287
}

(Dated: May, 2001)

\begin{abstract}
We demonstrate a concise method to enumerate the number of generalized polarizabilitiesquantities characterizing the independent observables in singly-virtual Compton scattering - for a target particle of arbitrary spin $s$. By using crossing symmetry and $J^{P C}$ conservation, we show that this number is $\left(10 s+1+\delta_{s, 0}\right)$.

PACS numbers: 13.88.+e, 13.60.Fz, 11.80.Cr, 11.30.Er
\end{abstract}

*Electronic address: Richard.Lebed@asu.edu 


\section{INTRODUCTION}

The process of virtual Compton scattering (VCS) off a target $t$ of spin $s$ offers new opportunities for probing the structure of $t$. In particular, the singly-virtual process $\gamma^{*}+t \rightarrow$ $\gamma+t$ obtained through $e^{-}+t \rightarrow e^{-}+\gamma+t$ is represented by more independent amplitudes than real Compton scattering (RCS) $\gamma+t \rightarrow \gamma+t$, owing to the additional longitudinal polarization of the virtual photon, and contains a richer dynamics than RCS since the virtual photon energy and momentum transfer are independent variables. Furthermore, the singlyvirtual process is experimentally feasible at laboratories such as Jefferson Lab, MIT-Bates, and MAMI.

The amplitudes for VCS are complicated to analyze because the real photon may couple either to the electron (Bethe-Heitler processes) or to the target $t$. In addition, there are trivial Born diagrams in which the intermediate state connecting the initial and final onshell target particles $t$ is just an off-shell copy of $t$; in such diagrams the internal structure of $t$ enters only through its elastic form factor at the vertices.

A theorem due to Low [1] proves that all photon scattering amplitudes from a target are regular once the Born terms have been subtracted, and indeed are at least of linear order in the real photon energy $\omega^{\prime}$. The observable coefficients of these linear terms characterize the scattering process in the limit of a soft final-state photon, and are called generalized polarizabilities (GPs). While the separation of GPs from Bethe-Heitler and Born amplitudes is a challenging problem of experimental analysis, the GPs themselves contain interesting physical information. The usual polarizabilities measure the response of a target to external electromagnetic fields, represented by scattering of photons with $q^{2}=0$. Generalized polarizabilities include $q$-dependence from the photon virtuality and thus are momentumspace quantities with the same relationship to the target polarization densities as the elastic charge form factor has to the target charge density (i.e., a Fourier transform into configuration space) [2].

The question of counting the independent GPs of a target of a given spin $s$ has been discussed in the literature for $s=0$ or $1 / 2$. The latter case of course includes nucleons, while the former describes the spin-averaged case for any $s$. Previously, the GPs were counted by constructing [3] the Compton amplitudes associated with a multipole expansion of the initial and final photons and matching the total $J^{P}$ eigenvalues of the asymptotic states. In particular, the authors of Ref. [3] found 3 GPs for the spin-averaged case and 10 for the full spin- $1 / 2$ case.

Subsequently, a direct calculation (Ref. 四) showed that only 2 of the 3 spin-averaged GPs are independent in the linear $\sigma$ model, raising suspicions that the GPs of Ref. [3] contain redundancies. In Ref. [5] the source of the over-counting was identified: The construction of Ref. [3] does not take into account the crossing symmetry (combined with charge conjugation symmetry) of the target $t$ in the process $\gamma^{*}+t \rightarrow \gamma+t$, and direct construction of the relevant tensors in the Compton scattering amplitude led to the expected 2 GPs in the spin-averaged channel. Subsequent work (Ref. [6]) by the same group showed that only 6 GPs are independent in the $s=1 / 2$ case.

The latter counting poses several interesting questions. First, is it obvious that the new construction does indeed take into account all the relevant symmetries? Second, this construction, while elegant, is still rather formidable and tends to obscure a direct understanding of why the counting of GPs turns out to give a particular number. Third, there is a very interesting numerical relation that holds for $s=0$ and $1 / 2$ : The number of GPs equals in each 
case (2 and 6, respectively) the number of amplitudes for RCS! We hasten to add that, even a priori, this is almost certainly a coincidence, since the GPs are associated with amplitudes linear in $\omega^{\prime}$ for an arbitrary $q^{2}$ in a fixed partial wave, while the real Compton amplitudes have $q^{2}=0$ and an arbitrary $\omega^{\prime}$, but sum over all allowed partial waves. If a one-to-one correspondence existed between these two disparate quantities, it would necessarily point to a highly subtle feature of the full Compton $S$-matrix.

In this short paper we seek to impose all relevant symmetries to the express end of enumerating the GPs for a target of arbitrary spin $s$. As in Refs. [5, 6] we find that crossing symmetry and charge conjugation symmetry are indeed essential ingredients to obtain the correct counting, and we render these symmetries manifest by performing the counting for the crossed process $\gamma^{*}+\gamma \rightarrow t+\bar{t}$. We obtain the same counting as these authors for $s=0$ and $1 / 2$. We also compute, for comparison, the number of amplitudes obtained for $\gamma^{*}+t \rightarrow \gamma+t$ through direct counting of partial waves in the multipole expansion (as in Ref. [3]), or equivalently, through helicity amplitudes: It is interesting to verify and extend the result of Ref. [3], and count the number of potential GPs eliminated by crossing symmetry. Finally, we perform a counting of helicity amplitudes in RCS - hardly a new problem, but included for completeness - and show that this number is larger than the number of GPs for targets with $s \geq 1$.

Apart from its intrinsic interest as a problem of separating purely symmetry-related and dynamical degrees of freedom, this counting proves useful if one wishes to measure the GPs of composite targets. For example, given the complete set of GPs of the deuteron (we show that there are 11) and the 6 of the proton, it is possible to obtain interesting information on the electromagnetic structure of the neutron.

\section{GP COUNTING WITHOUT CROSSING CONSTRAINTS}

\section{A. The Multipole Basis}

We begin by noting the central constraint that makes the counting of GPs different from that of full helicity amplitudes, namely, that the final-state photon is assumed soft. In particular, this implies that only the $\mathrm{E} 1\left(1^{-}\right)$and $\mathrm{M} 1\left(1^{+}\right)$multipoles are permitted, since they alone have wave functions for transversely polarized photons that vary linearly with the photon energy $\omega^{\prime}$; the higher multipoles are at least quadratic in $\omega^{\prime}$. Thus, only a limited set of relative angular momenta between the final-state $t$ and $\gamma$ are allowed, which are already included in the multipole wave function.

To be explicit, let us work in the center of momentum (CM) frame of the process $\gamma^{*}(q)+$ $t(p) \rightarrow \gamma\left(q^{\prime}\right)+t\left(p^{\prime}\right)$, which we call the $s$ channel. Without loss of generality, the momentum components in this frame (using the notation of Refs. [5, 6]) may be labeled

$$
\begin{aligned}
q^{\mu} & =\left(\omega_{0}, 0,0,+\bar{q}\right), \\
p^{\mu} & =(E, 0,0,-\bar{q}), \\
q^{\prime \mu} & =\left(\omega^{\prime},+\omega^{\prime} \sin \theta, 0,+\omega^{\prime} \cos \theta\right), \\
p^{\prime \mu} & =\left(E^{\prime},-\omega^{\prime} \sin \theta, 0,-\omega^{\prime} \cos \theta\right)
\end{aligned}
$$

where $E=\sqrt{\bar{q}^{2}+m_{t}^{2}}, E^{\prime}=\sqrt{\omega^{\prime 2}+m_{t}^{2}}$, and overall energy conservation requires $\omega_{0}=$ $E^{\prime}+\omega^{\prime}-E$. Let us also define $P=p+p^{\prime}$. There are clearly three independent kinematic 
variables, whether labeled as $\omega^{\prime}, \bar{q}$, and $\theta$, or the invariants $q^{2}, q \cdot q^{\prime}$, and $q \cdot P$, or the Mandelstam variables $s, t$, and $u$ (note that $s+t+u$ is not fixed since the virtual photon does not have a fixed mass). In particular, one finds

$$
\omega^{\prime}=\frac{q \cdot q^{\prime}+q \cdot P}{2 \sqrt{m_{t}^{2}+q \cdot q^{\prime}+q \cdot P}}=\frac{s-m_{t}^{2}}{2 \sqrt{s}} .
$$

The counting of allowed partial wave amplitudes in the $s$ channel may be carried out either using the multipole expansion or helicity amplitudes, and we now demonstrate how each is accomplished.

To find the total $J^{P}$ of a given final state, one need only add the E1 or M1 photon to the target spin-parity, $s^{\Pi}$. These values are $J^{P}=\{|s-1|, \ldots, s+1\}^{-\Pi}$ and $\{|s-1|, \ldots, s+1\}^{\Pi}$, respectively. These sets contain 3 values of $J$ if $s \geq 1,2$ if $s=1 / 2$, and 1 if $s=0$, and so it is convenient to treat the exceptional cases $s=0$ and $1 / 2$ explicitly. The initial virtual photon may be in any multipole (including the longitudinal Coulomb modes) for which adding the initial target spin-parity $s^{\Pi}$ gives the same total $J^{P}$. For example, if $s^{\Pi}=1^{+}$ and the final photon is E1, then $J^{P}=0^{-}, 1^{-}, 2^{-}$. Among the initial allowed multipoles is M2 $\left(2^{-}\right)$, which added to $1^{+}$gives $1^{-}, 2^{-}, 3^{-}$. Thus one finds two allowed amplitudes for an initial M2 photon and final E1 photon, corresponding to $J^{P}=1^{-}, 2^{-}$. By the triangle rule of angular momentum addition, the largest allowed multipole rank $n$ satisfies $n-s=s+1$, or $n=2 s+1$.

Since allowing both E1 and M1 photons includes both parities, generic values of $J$ permit an equal number of amplitudes coupled to $\mathrm{E} n, \mathrm{M} n$, and $\mathrm{C} n$. The exception is for $n=0$, since there are no $\mathrm{E} 0$ or $\mathrm{M} 0$ multipoles but only $\mathrm{C} 0\left(0^{+}\right)$. In that case, the only potentially allowed amplitude has $J^{P}=s^{\Pi}$, which is always permitted by the final state unless $s=0$. Thus there are $\left(1-\delta_{s, 0}\right)$ amplitudes with initial C0 photons. All other values of $n$ give 3 amplitudes. The total number is therefore

$$
\left(1-\delta_{s, 0}\right)+3 \sum_{n=1}^{2 s+1}[\text { Overlaps between }\{|n-s|, \ldots, n+s\} \text { and }\{|s-1|, \ldots, s+1\}] \text {. }
$$

It is straightforward to count the terms in the sum by dividing them into cases. The members of the set $\{|s-1|, \ldots, s+1\}$ are distinct if $s \geq 1$, leading to 3 overlaps for $n \leq 2 s-1,2$ for $n=2 s$, and 1 for $n=2 s+1$; the special cases $s=0$ and $1 / 2$ may be handled separately. Carrying this out, one finds the number of amplitudes in the $s$ channel to be

$$
18 s+1+2 \delta_{s, 0} .
$$

We hasten to add that this is the counting of amplitudes not taking into account the crossing symmetry of the initial and final $t$ particles. Only $J^{P}$ conservation and the exhaustion of $O\left(\omega^{\prime}\right)$ amplitudes by E1 and M1 multipoles has been used. This is, in fact, a counting equivalent to that done in Ref. [3], that of counting the number of coefficients of terms linear in $\omega^{\prime}$, identified in Ref. [3] as the GPs: The cases $s=0$ and $1 / 2$ give 3 and 10, respectively.

\section{B. The Helicity Amplitude Basis}

One might worry that the multipole expansion is relevant only nonrelativistically (since

$\omega^{\prime}$ is treated as a perturbative parameter). However, the multipoles simply count quantum 
numbers and therefore the corresponding amplitudes are fully relativistic. Put another way, the true multipole amplitudes begin with a certain power of $\omega^{\prime}$ as determined by their low-energy behavior, but contain higher powers as well. One can verify this statement explicitly by employing a manifestly relativistic formalism, the helicity decomposition [4, 8]. The counting procedure is very similar to that performed in Ref. [9]. Define $\psi_{J M \lambda_{1} \lambda_{2}}$ as a two-particle helicity state in the CM. Angular momentum conservation along the CM axis requires $\left|\lambda_{1}-\lambda_{2}\right| \leq J$. With $\eta_{1,2}$ being the intrinsic parities of the two particles, the action of the parity operator is

$$
\hat{P} \psi_{J M \lambda_{1} \lambda_{2}}=\eta_{1} \eta_{2}(-1)^{J} \psi_{J M-\lambda_{1}-\lambda_{2}}
$$

meaning that the parity \pm eigenstates are given by

$$
\psi_{J M \lambda_{1} \lambda_{2}} \pm \eta_{1} \eta_{2}(-1)^{J} \psi_{J M-\lambda_{1}-\lambda_{2}} .
$$

It is not necessary to carry out a helicity decomposition of the final state since the restriction to the E1 and M1 multipoles already determines the allowed values of $J^{P}$; indeed, the assumption of a soft photon already lies beyond the scope of the helicity decomposition, which is completely general since it allows any relative angular momentum. On the other hand, one may study the initial state of $\gamma^{*}+t$ in terms of helicity basis partial waves instead of using the multipole basis as in the previous counting. The intrinsic parity of the virtual photon is -1 ; only the $1^{-}$polarizations need be considered, since the $0^{+}$polarization may be eliminated through current conservation. Thus, the product $\eta_{1} \eta_{2}$ in Eq. (6) equals $-\Pi$. Moreover, the two terms in Eq. (6) are distinct, so that both parity eigenstates survive, unless $\lambda_{1}=\lambda_{2}=0$, in which case only one parity eigenstate survives for each value of $J$.

Now the physical input is complete, and one need only enumerate possible values of $\lambda$ to count amplitudes. Since each combination in Eq. (6) includes both the $\lambda_{1}, \lambda_{2}$ and $-\lambda_{1},-\lambda_{2}$ combinations, it is completely general to consider only the two types of combinations $(0<$ $\left.\lambda_{1} \leq s_{1},-s_{2} \leq \lambda_{2} \leq s_{2}\right)$ or $\left(\lambda_{1}=0\right.$ [if 1 is a boson], $\left.0 \leq \lambda_{2} \leq s_{2}\right)$. In the present case, let 1 be the virtual photon so that $s_{1}=1$, and 2 be the target so that $s_{2}=s$. Then the two cases are $\left(\lambda_{\gamma^{*}}=+1,-s \leq \lambda_{t} \leq s\right)$ and $\left(\lambda_{\gamma^{*}}=0, \lambda_{s} \geq 0\right)$.

If $\lambda_{\gamma^{*}}=+1$, then the restriction on $J$ reads $\left|1-\lambda_{t}\right| \leq J \in\{|s-1|, s, s+1\}$. We have seen that the parity eigenvalue does not matter here; if $\lambda_{t}$ is allowed then both parities appear. The special cases $s=0$ and 1/2 may again be handled separately, so that the members of $\{s-1, s, s+1\}$ are distinct. Then, by dividing into classes based on whether $\left|1-\lambda_{t}\right| \leq s-1$ (leading to 3 amplitudes), or $\left|1-\lambda_{t}\right|=s$ (2 amplitudes), or $\left|1-\lambda_{t}\right|=s+1$ (1 amplitude), one finds a total of $12 s$ amplitudes, including parity doubling.

For $\lambda_{\gamma^{*}}=0$, only $\lambda_{t} \geq 0$ need be considered, and only $\lambda_{t} \neq 0$ receives a parity doubling. Again handling $s=0$ and $1 / 2$ separately, one may take $s \geq 1$. Then the restriction on $J$ reads $\lambda_{t} \leq J \in\{s-1, s, s+1\}$, leading to 3 amplitudes $\left(\lambda_{t} \leq s-1\right)$ or 2 amplitudes $\left(\lambda_{t}=s\right)$. With the floor function denoted as usual by square brackets, there are $[s+1 / 2]$ values of $\lambda_{t}>0$ and $\left[1+(-1)^{2 s}\right] / 2$ values of $\lambda_{t}=0$, and weighting these by the parity multiplicities, one finds a total of $6 s+1$ amplitudes [all factors of $(-1)^{2 s}$ cancel]. Combining this with the $\lambda_{\gamma^{*}}=+1$ amplitudes and including the special cases of $s=0$ and $1 / 2$ (which give the same number of amplitudes as before), one again finds a total of $\left(18 s+1+2 \delta_{s, 0}\right)$ amplitudes. 


\section{GP COUNTING USING CROSSING CONSTRAINTS}

As mentioned above, the $s$ channel is not the channel of highest symmetry because there are constraints due to the crossing symmetry that have not been taken into account. We accomplish this by considering instead the $t$ channel process $\gamma^{*}(q)+\gamma\left(-q^{\prime}\right) \rightarrow t\left(p^{\prime}\right)+\bar{t}(-p)$ (the double use of $t$ as a kinematic variable and the target particle should not confuse the reader). The signs on the momenta are designed to allow the same notation for all dot products in the $S$ matrix regardless of which crossed channel one considers. That is, $p+q=p^{\prime}+q^{\prime}$ in all channels.

It is again convenient in the helicity decomposition to consider the process in the CM. The momentum components may be labeled

$$
\begin{aligned}
q^{\mu} & =\left(q_{0}, 0,0,+\omega\right), \\
-q^{\prime \mu} & =(\omega, 0,0,-\omega), \\
p^{\prime \mu} & =\left(\bar{E}^{\prime},-\rho \sin \phi, 0,-\rho \cos \phi\right), \\
-p^{\mu} & =(\bar{E},+\rho \sin \phi, 0,+\rho \cos \phi),
\end{aligned}
$$

where $\bar{E}=\sqrt{\rho^{2}+m_{t}^{2}}=\bar{E}^{\prime}$ and energy conservation gives $q_{0}+\omega=2 \bar{E}$. The three independent kinematic variables in this frame may be taken as $\omega, \rho$, and $\phi$, and one finds

$$
\omega=\frac{-q \cdot q^{\prime}}{\sqrt{q^{2}-2 q \cdot q^{\prime}}}=\frac{2 m_{t}^{2}-s-u}{2 \sqrt{t}} .
$$

In both the $s$ and $t$ channels the real photon is assumed soft. However, this implies in the $s$ channel $\mathrm{CM}$ that the final $t$ has a small spatial momentum and in the $t$ channel that the $\gamma^{*}$ has a small spatial momentum. The two frames have different kinematics [as is apparent from comparing Eqs. (2) and (8)], so how can it be that there is a relationship between amplitudes linear in $\omega^{\prime}$ and those linear in $\omega$ ? The answer is that the transformation between $\omega^{\prime}$ and $\omega$, whether written in terms of the other two kinematic variables of the $s$ channel or the $t$ channel, preserves the linearity in either variable. Explicitly,

$$
\omega=-\omega^{\prime} \frac{\left(E^{\prime}-E+\omega^{\prime}-\bar{q} \cos \theta\right)}{\sqrt{2\left(m_{t}^{2}-E^{\prime} E+\bar{q} \omega^{\prime} \cos \theta\right)}} .
$$

This linear-to-linear mapping arises from the masslessness of the real photon, and in particular that all components of $q^{\prime}$ vanish like $\omega^{\prime}$ or $\omega$ in the soft photon limit. It implies that amplitudes linear in soft photon energies may be regarded as GPs in either crossing, and that the process with the smaller number of such amplitudes (call this the minimal process) provides the correct counting of independent GPs. The extra amplitudes in other crossings must therefore be redundant precisely because of the ignored constraints of crossing symmetry and the application of additional symmetries manifest only in the minimal process.

Two points should be clarified before proceeding. First, the parameters $\omega$ or $\omega^{\prime}$, while each defined in the relevant CM frame, actually represent two different functions of Lorentz invariant quantities [as apparent from Eqs. (2) and (8)]. They both become small in the soft photon limit because two independent Lorentz invariants, $q \cdot q^{\prime}$ and $q \cdot P$, or equivalently $s-m_{t}^{2}$ and $u-m_{t}^{2}$, become small in this limit; the coefficients of both contribute to the GPs. Second, we note that the transformation Eq. (9) is pure imaginary since the argument 
of the denominator square root is negative for real allowed values of $\bar{q}, \omega^{\prime}$, and $\theta$; this simply indicates that fixed values for the three invariants that give real kinematics in the $s$ channel do not give real kinematics in the $t$ channel. But the existence of a certain number of amplitudes or GPs should not depend on the reality of the momentum components, since the full amplitudes are complex analytic functions of the invariants.

We now argue that the $t$ channel represents the minimal process. We have already noted that the $s$ channel possesses a crossing symmetry between the initial and final $t$ not yet properly taken into account. In the $t$ channel this symmetry is translated into charge conjugation symmetry of the $t+\bar{t}$ system; the $\gamma^{*}+\gamma$ system always has $C=(-1)^{2}=+1$. $C$ invariance is of course not applicable in the $s$ channel unless $t$ is self-conjugate. There is clearly no remaining crossing symmetry to take into account in the $t$ channel, nor $T$ invariance, since the initial and final states consist of completely distinct particles. Thus, the only symmetry restrictions applicable to the $t$ channel are $J^{P C}$ conservation.

As before, we work in the helicity formalism. The action of charge conjugation on a self-conjugate two-particle state gives

$$
\hat{C} \psi_{J M \lambda_{1} \lambda_{2}}=(-1)^{J} \psi_{J M \lambda_{2} \lambda_{1}}
$$

Combining Eqs. (5) and (10) and suppressing the $J M$ subscripts, one obtains the $J^{P C}$ eigenstates

$$
\begin{array}{ll}
P C=++: & \psi_{\lambda_{1} \lambda_{2}}+(-1)^{J} \psi_{\lambda_{2} \lambda_{1}}+(-1)^{J} \psi_{-\lambda_{1}-\lambda_{2}}+\psi_{-\lambda_{2}-\lambda_{1}}, \\
P C=+-: & \psi_{\lambda_{1} \lambda_{2}}-(-1)^{J} \psi_{\lambda_{2} \lambda_{1}}+(-1)^{J} \psi_{-\lambda_{1}-\lambda_{2}}-\psi_{-\lambda_{2}-\lambda_{1}}, \\
P C=-+: & \psi_{\lambda_{1} \lambda_{2}}+(-1)^{J} \psi_{\lambda_{2} \lambda_{1}}-(-1)^{J} \psi_{-\lambda_{1}-\lambda_{2}}-\psi_{-\lambda_{2}-\lambda_{1}}, \\
P C=--: & \psi_{\lambda_{1} \lambda_{2}}-(-1)^{J} \psi_{\lambda_{2} \lambda_{1}}-(-1)^{J} \psi_{-\lambda_{1}-\lambda_{2}}+\psi_{-\lambda_{2}-\lambda_{1}} .
\end{array}
$$

As before, the soft photon appears only in the multipoles E1 and M1; however, note that the relative angular momentum in this case is measured with respect to the $\gamma^{*}$ rather than the $t$. The allowed $J^{P C}$ values are then $(0,1,2)^{++}$for $\mathrm{E} 1$ and $(0,1,2)^{-+}$for M1. For the $t+\bar{t}$ combination, therefore, one need consider only the $J^{-+}$and $J^{++}$combinations.

The physics input now being complete, let us review the ingredients before proceeding with the counting. As before, start with $J^{P}$ conservation and the observation that only E1 and M1 multipoles give CM amplitudes linear in $\omega^{\prime}$, and hence GPs. Then note that the original ( $s$-channel) process possesses a crossing symmetry due to the on-shell $t$ in both initial and final states. Considering instead the corresponding $t$-channel process, one finds that the original crossing symmetry is no longer manifest; but unlike in the $s$ channel, one may classify amplitudes by eigenvalues of charge conjugation: Effectively, crossing symmetry has been traded for $C$ symmetry. To complete the argument, it must be checked that GPs in the $s$ channel (coefficients of amplitudes linear in $\omega^{\prime}$ ) map to what may be called the GPs in the $t$ channel CM (coefficients of amplitudes linear in $\omega$ ); this is verified explicitly by Eq. (9). Since $J^{P C}$ conservation accounts for all relevant symmetries in the $t$ channel, the GPs thus obtained must represent the minimal set of GPs, even in the original $s$ channel.

As before, angular momentum conservation along the helicity axis requires $\left|\lambda_{1}-\lambda_{2}\right| \leq J$. It is convenient to separate into the cases $\lambda_{1}=\lambda_{2}, \lambda_{1}=-\lambda_{2}$, and all other pairs $\left(\lambda_{1}, \lambda_{2}\right)$. If $\lambda_{1}=\lambda_{2} \equiv \lambda$, then the $P C=++$ and $P C=-+$ cases in Eq. (11) simplify to

$$
\left[\psi_{\lambda \lambda}+\psi_{-\lambda-\lambda}\right]\left[1+(-1)^{J}\right],\left[\psi_{\lambda \lambda}-\psi_{-\lambda-\lambda}\right]\left[1+(-1)^{J}\right]
$$


respectively, meaning that each is allowed only for $J$ even. The case $\lambda_{1}=-\lambda_{2} \equiv-\lambda$ is distinguished only for $J^{-+}$, which according to Eq. (11) vanishes identically. All other $C=+$ helicity amplitudes are allowed for either parity as long as $\left|\lambda_{1}-\lambda_{2}\right| \leq J$.

It then becomes a straightforward exercise in counting the values of $\lambda_{1}, \lambda_{2}$ satisfying these conditions, and due to the form of Eq. (11) one may take $\lambda_{1} \geq 0$ without loss of generality. Table If summarizes the $t+\bar{t}$ helicity constraint on each $J^{P \bar{C}}$ and the number of allowed pairs $\left(\lambda_{1}, \lambda_{2}\right)$, which equals the number of allowed amplitudes. In cases where more than one value of $\left|\lambda_{1}-\lambda_{2}\right|$ is allowed, the number of amplitudes has been expanded to show how many occur for each value. All that remains to obtain a final answer is to note that $[j]=j-\left[1-(-1)^{2 j}\right] / 4$ for $j$ integral or half-integral. Summing the cases, one finds

$$
\text { Number of independent GPs }=10 s+1+\delta_{s, 0} .
$$

Thus, $8 s+\delta_{s, 0}$ of the GPs in the counting leading to Eq. (四) are redundant due to crossing symmetry.

\section{COUNTING RCS HELICITY AMPLITUDES}

The final counting we perform is that of helicity amplitudes of RCS for a target of spin $s$. This again is carried out most conveniently in the $t$ channel using $J^{P C}$ invariance (it can also be done in the $s$ channel, where one must then impose time reversal invariance since the initial and final on-shell photons are identical). Helicity amplitudes sum over many partial waves, meaning that the precise value of $J$ is irrelevant to us as long as it is equal in the initial and final states. However, as is apparent from Eq. (11), it gives different linear combinations depending on whether $J$ is even or odd, and these correspond to distinct helicity states. This is apparent from the discussion surrounding Eq. (12): Since real photons have only $\lambda= \pm 1$, there are two 2-photon helicity states with $J^{++}$even, $\lambda_{1}=\lambda_{2}=+1$ and $\lambda_{1}=-\lambda_{2}=+1$, while only the latter is allowed for $J^{++}$odd. The $J^{-+}$two-photon combination vanishes for $\lambda_{1}=-\lambda_{2}=+1$, while for $\lambda_{1}=\lambda_{2}=+1$ it survives only for even $J$.

To count the allowed $t+\bar{t}$ helicity combinations, we note that it is sufficient to consider the two cases $\left(\lambda_{1}>0\right.$ and $\left.\lambda_{2} \geq 0\right)$, which gives $[s+1 / 2][s+2]$ combinations, or $\left(\lambda_{1}=\lambda_{2}=0\right)$, which gives $\left[1+(-1)^{2 s}\right] / 2$ combinations. Again using the reasoning around Eq. (12), if $P C=++$, then the first case always allows $J$ even, and allows $J$ odd except when $\lambda_{1}=\lambda_{2}$, which occurs in $[s+1 / 2]+\left[1+(-1)^{2 s}\right] / 2$ combinations. If $P C=-+$, then $J$ odd is not allowed since the corresponding two-photon state vanishes, while $J$ even is allowed unless $\lambda_{1}=-\lambda_{2}$, which occurs in $[s+1 / 2]+\left[1+(-1)^{2 s}\right] / 2$ combinations. Matching with the allowed two-photon states, one finds a total of

$$
\begin{aligned}
& 4\left\{[s+1 / 2][s+2]+\left[1+(-1)^{2 s}\right] / 2\right\}-2\left\{[s+1 / 2]+\left[1+(-1)^{2 s}\right] / 2\right\} \\
= & 2(s+1)(2 s+1)
\end{aligned}
$$

RCS helicity amplitudes. It is amusing to note that this number is greater than the number of GPs in Eq. (13) by an amount $(2 s-1)^{2}-\delta_{s, 0}$, a perfect square that vanishes for $s=0$ and $1 / 2$ but not for $s \geq 1$.

In summary, we have shown that the number of independent generalized polarizabilities for Compton scattering on a target of spin $s$ is $\left(10 s+1+\delta_{s, 0}\right)$, that the number of multipole

amplitudes in the soft photon limit, not taking into account the crossing symmetry of the 
TABLE I: Constraints on $t+\bar{t}$ helicities and number of amplitudes for allowed values of $J^{P C}$.

\begin{tabular}{ccc}
\hline \hline $0^{++}$ & $\lambda_{1}=\lambda_{2}$ & {$[s+1]$} \\
$1^{++}$ & $\left|\lambda_{1}-\lambda_{2}\right|=1$ & {$[s+1 / 2]$} \\
$2^{++}$ & $\left|\lambda_{1}-\lambda_{2}\right|=0,1,2$ & {$[s+1]+[s+1 / 2]+[s]$} \\
$0^{-+}$ & $\lambda_{1}=\lambda_{2} \neq 0$ & {$[s+1 / 2]$} \\
$1^{-+}$ & $\left|\lambda_{1}-\lambda_{2}\right|=1, \lambda_{1} \neq-\lambda_{2}$ & {$[s]$} \\
$2^{-+}$ & $\left|\lambda_{1}-\lambda_{2}\right|=0,1,2$ & {$[s+1 / 2]+[s]+\left([s-1 / 2]+\delta_{s, 0}\right)$} \\
\hline \hline
\end{tabular}

target, is $\left(18 s+1+2 \delta_{s, 0}\right)$, and that the number $2(s+1)(2 s+1)$ of real Compton scattering helicity amplitudes exceeds the number of generalized polarizabilities for $s \geq 1$ and is equal in the cases $s=0$ and $1 / 2$ previously studied.

\section{Acknowledgments}

I would like to thank Richard Jacob and Xiangdong Ji for valuable criticisms and illuminating conversations. This work was supported in part by the Department of Energy under grant No. DOE-AC05-84ER40150.

[1] F.E. Low, Phys. Rev. 96, 1428 (1954).

[2] B.R. Holstein, hep-ph/0010129, presented at Chiral Dynamics 2000, Newport News, Virginia, July, 2000 (to appear in Proceedings).

[3] P.A.M. Guichon, G.Q. Liu, and A.W. Thomas, Nucl. Phys. A591, 606 (1995).

[4] A. Metz and D. Drechsel, Mainz Report No. MKPH-96-17, nucl-th/9607050, presented at Workshop on Virtual Compton Scattering, Clermont-Ferrand, France, June, 1996.

[5] D. Drechsel, G. Knöchlein, A. Metz, and S. Scherer, Phys. Rev. C 55, 424 (1997).

[6] D. Drechsel, G. Knöchlein, A. Yu. Korchin, A. Metz, and S. Scherer, Phys. Rev. C 57, 941 (1998).

[7] M. Jacob and G.C. Wick, Ann. Phys. 7, 404 (1959).

[8] V. B. Berestetskii, E. M. Lifshitz, and L. P. Pitaevskii, Quantum Electrodynamics, Pergamon, Oxford, England, 1982.

[9] X. Ji and R.F. Lebed, Phys. Rev. D 63, 076005 (2001). 